\title{
Revision of the Neotropical spider genus Itatiaya Mello-Leitão (Araneae, Ctenidae) with considerations on biogeographic distribution of species
}

\author{
Daniele Polotow \& Antonio D. Brescovit
}

Laboratório de Artrópodes, Instituto Butantan. Avenida Vital Brazil 1500, 05503-900 São Paulo, São Paulo, Brasil. E-mail: adbresc@terra.com.br; danielepolotow@butantan.gov.br.

\begin{abstract}
Itatiaya Mello-Leitão, 1915, is revised and the type-species I. modesta Mello-Leitão, 1915, is redescribed and considered a senior synonym of Centroctenus sai Brescovit, 1996. Seven species are newly described: Itatiaya

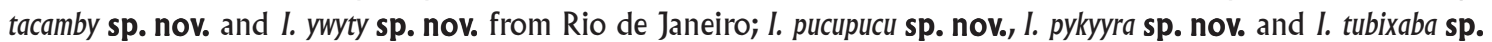
nov., from Minas Gerais; I. iuba sp. nov. from São Paulo; l. apipema sp. nov. from São Paulo and Rio de Janeiro. Notes on their distribution from Brazilian Atlantic Forest are presented.

KEY WORDS. Atlantic Forest; Brazil; distribution; systematics.
\end{abstract}

RESUMO. Revisão das aranhas neotropicais do gênero Itatiaya Mello-Leitão (Araneae, Ctenidae). O gênero Itatiaya Mello-Leitão, 1915, é revisado e sua espécie-tipo, I. modesta Mello-Leião, 1915, é redescrita e considerada um sinônimo senior de Centroctenus sai Brescovit, 1996. Sete espécies são descritas: Itatiaya tacamby sp. nov. e I. ywyty sp. nov. do Rio de Janeiro; I. pucupucu sp. nov., l. pykyyra sp. nov. e I. tubixaba sp. nov., de Minas Gerais; I. iuba sp. nov. de São Paulo; I. apipema sp. nov. de São Paulo e Rio de Janeiro. Notas sobre sua distribuição na Floresta Atlântica brasileira são apresentadas.

PALAVRAS-CHAVE. Brasil; distribuição; Floresta Atlântica; sistemática.

The genus Itatiaya was proposed by Mello-Leitão (1915), to include I. modesta Mello-Leitão, 1915, described from Parque Nacional do Itatiaia, Resende, Rio de Janeiro, Brazil, based on a single female specimen. The holotype should be deposited in the collection of the Museu Nacional, Rio de Janeiro, but was not located and is probably lost. The original description includes no illustrations and lists only few diagnostic characters. The lost holotype and this brief description resulted in doubts about the status of this genus. These doubts arise especially because of the eye arrangement description, that differs from the general ctenid pattern, with the anterior lateral eyes equally distant from the anterior median eyes and the posterior median eyes. Nevertheless, this same pattern is observed in species of the genus Asthenoctenus Simon (1897), revised by Siмó \& EICKSTEDT (1994).

In the original description, Itatiaya Mello-Leitão, 1915, was listed as a member of Cteninae, Clubionidae. Petrunkevitch (1928) elevated Cteninae to family rank, and the genus was then listed in Cteninae, Ctenidae. Mello-Leitão (1936) presented a revision of Ctenidae and its subfamilies, including the genus Itatiaya in Cteninae. Later, the genus was transferred to Sparassidae by BRYANT (1942), and listed in the subfamily Micrommatinae. Lehtinen (1967) returned Itatiaya to Ctenidae, and cited it as incertae sedis. SILVA (2003) presented a cladistic analysis for the family Ctenidae, but Itatiaya was not included in the analysis because of the lack of material in collections. Nevertheless, SILVA (2003) suggests that Itatiaya could be a member of the Odo clade (Zoridae), based on the original description. However, in the most recent version of the spider catalogue, Platnick (2005) lists Itatiaya in Ctenidae.

In addition to the type species, two other species were included in Itatiaya, but were later transferred to other Ctenidae genera: Itatiaya amica Mello-Leitão, 1941, transferred to Asthenoctenus, and considered a junior synonym of $A$. borellii Simon, 1897 by SIMÓ \& EICKSTEDT (1994); and Itatiaya isolata Bryant, 1942, transferred to Trujillina Bryant, 1948, by LeHTINEN (1967). Until the present revision, the genus remained monotypic, including only $I$. modesta.

Recently, several samplings were carried out in mountainous regions in southeastern Brazil by the project "Biodiversity of Arachnida and Myriapoda of the state of São Paulo" of the BIOTA/FAPESP program. These samplings resulted in a great number of specimens of the genus Itatiaya. These specimens were identified as Itatiaya based mainly on two diagnostics characters listed in the original description: the position of the lateral anterior eyes and depressions on the lateral fields of the female epigynum. Itatiaya modesta, the type species, was recognized by the charaters cited above and by the fact that the specimens were collected in the type locality, enabling the redescription of the genus and the proposal of a neotype.

Revista Brasileira de Zoologia 23 (2): 429-442, junho 2006 
In this paper, Itatiaya is revised and the type species $I$. modesta is redescribed and considered a senior synonym of Centroctenus sai Brescovit, 1996. In addition, seven new species are described. Notes on their distribution from Brazilian high mountain Atlantic Forest are presented.

\section{MATERIAL AND METHODS}

The material examined belongs to the following institutions (curators in parenthesis): IBSP, Instituto Butantan, São Paulo (A. D. Brescovit); MZSP, Museu de Zoologia, Universidade de São Paulo, São Paulo (R. Pinto da Rocha); MNRJ, Museu Nacional do Rio de Janeiro, Rio de Janeiro (A.B. Kury). All measurements are in milimiters. Descriptions and measurements follow BRESCOVIT (1996). Micrographs were obtained with a JEOL scanning electron microscope (JSM 840A) from the "Laboratório de Microscopia Eletrônica do Departamento de Física Geral, Instituto de Física, Universidade de São Paulo (USP)". Abbreviations: (RTA) retrolateral tibial apophysis, (AME) anterior median eyes, (ALE) anterior lateral eyes, (PME) posterior median eyes, (PLE) posterior lateral eyes, $(\mathrm{p})$ prolateral, $(\mathrm{r})$ retrolateral, $(\mathrm{v})$ ventral.

\section{Itatiaya Mello-Leitão, 1915}

Itatiaya Mello-Leitão, 1915: 139 (Type species Itatiaya modesta Mello-Leitão, 1915, by monotypy); Petrunkevitch, 1928: 147; Mello-Leitão, 1936: 1; Bryant, 1942: 9; Bonnet, 1961: 267; Lehtinen, 1967: 241; Brignoli, 1983: 589; Silva, 2003: 28; Platnick, 2005.

Diagnosis. Species of Itatiaya resemble those of Asthenoctenus by the two recurved eye rows and the position of ALE distant from PME, but differ from this and the remaining ctenid genera by the combination of the following characters: cymbium with extensive dorsal scopulae (Fig. 28), except I. pucupucu sp. nov.; male palpal tibia with large, uniquely modified RTA and a slightly rounded subventral process covered by long hairs (Fig. 27), except I. puсириси sp. nov.; median apophysis often pointed distally (Fig. 27); epigynum with large median plate and lateral field excavated (Figs 36-37) and internally, spermathecae with a median constriction (Fig. 38).

Description. Small ecribellate ctenids, with total length (male and female) ranging from 4.00 to 11.00. Sexual dimorphism not evident, but male carapace generally large and female generally more robust (Figs 25, 26). Carapace piriform; widest between legs II and III, truncated posteriorly. Thoracic groove longitudinal, long and deep, in the posterior third of the carapace. Eye arrangement in three rows, with the second row procurved, similar to Asthenoctenus (Simó \& EICKSTEDT 1994: fig. 1), with the ALE separated from AME, by at least one time AME diameter. Clypeus with long and erected black bristles. Eye diameters: ALE larger than AME; PME and PLE proportional in size, and both larger than ALE and AME. Eye interdistances: AME separated by slightly less than half their diameter, PME-PLE slightly less than AME diameter, ALE-PLE by the diameter of
ALE, AME-PME by slightly less than half of AME diameter, AMEALE by the third part of the AME diameter. Clypeal height generally proportional or slightly larger than AME diameter. Chelicerae dark brown with 3 promarginal and 3 retromarginal teeth (Fig. 9); cheliceral condyle often conspicuous, sometimes reduced. Chilum divided and elongated laterally. Endites longer than labium, convex in the lateral border, truncated distally, serrula with 15-20 tiny teeth, with a small scopulae on the distal area and long bristles in the internal border. Labium subquadrangular, truncated at the apex, with long bristles. Sternum rounded, anteriorly truncated, with sinuous lateral borders, not projected between coxae IV. Legs slender in the male and generally more robust in the female. Tibiae I and II with five pair of ventral spines (Fig. 1) and metatarsus I and II with three pairs of ventral spines. Leg formula 4123 or 4132 . Leg spination: tibia I II v2-2-2-2-2, p0-1-1, r0-1-1, III v2-2-2, p0-1-1-0, r1-1-1-0, IV v22-2, p0-1-1-0, r0-1-1-0, metatarsus I v2-2-2-1v, p1-1-1, r1-1-1, II v2-2-2-1v, p0-1-1, r1-1-1-1, III v2-2-2, p0-1-1, r1-1-1, IV v2-2-22, p1-1-1-1, r1-1-1. Trochanters I-IV notched. Males with sparce scopulae on tarsi and base of metatarsi, dense in females. Tarsus with two claws with 6-7 teeth and dense claw tufts (Figs 4, 5). Tarsal organ distal and at least with 5-6 chemeoreceptors (Fig. 2 ). Trichobothria with four to five transversal grooves at dorsal plate (Fig. 3). Female pedipalp hirsute, with ventral scopulae with long hairs and short claw with 5 teeth (Fig. 10). Abdomen oval, with black bristles on anterior border and two pairs of anterior median dorsal muscular impressions (Figs 25, 26). Palpal tibia elongated, at least two times longer than cymbium. Cymbium oval with extensive dorsal scopulae (Figs 12, 13, 28), except $I$. рисириси sp. nov. Generally with large retrolateral tibial apophysis (except I. pucupucu sp. nov.), with a subventral rounded apophysis of RTA with long hairs, and with a short ventral projection (Fig. 27). RTA bifid, with internal branch short and rounded, and external branch single, double or with several points (Fig. 27). Median apophysis large and curved, often apically pointed. Conductor membranous, elongate and generally wrapping the embolic tip. Embolus slender with a large base. Epigynum formed by a median plate, excaveted lateral fields, ventral lateral spurs and dorsal lateral spurs (Fig. 36). Internally with copulatory ducts elongated. Spermathecae with median constriction with postero-ventral fertilization ducts (Fig. 38). Anterior spinnerets with at least 30 cilindrical spigots (Fig. 6). Median and posterior spinnerets with three large cilindrical spigots and aproximately 30-40 piriform gland spigots (Figs 7, 8).

Composition. Eight species.

Distribution. Known from southeastern of Brazil.

\section{Itatiaya modesta Mello-Leitão, 1915 Figs 1-5, 9-16, 25-39}

Itatiaya modesta Mello-Leitão, 1915: 139 (female holotype from Brazil, Rio de Janeiro: Resende, Parque Nacional do Itatiaia, not examined, should be deposited in MNRJ, not found, probably lost); Petrunkevitch, 1928: 147; Mello-Leitão, 1936: 
1; Bryant, 1942: 9; Bonnet, 1961: 267; Lehtinen, 1967: 241; Brignoli, 1983: 589; Platnick, 2005.

Centroctenus sai Brescovit, 1996: 312, figs 37, 38 (male holotype from Brazil, São Paulo: Cotia, IV.1982, H.B. Sá leg., deposited in IBSP 4961, examined; female paratype from BRAZIL, São Paulo: São Paulo, VIII.1987, M.C. Laranjeira leg. deposited in IBSP 4178, examined); Platnick, 2005. Syn. nov.

Diagnosis. Itatiaya modesta resembles I. tacamby sp. nov. by the morphology of the embolus and conductor, but differs by the morphology of the external branch of the RTA and by the non bifid median apophysis in the male palp (Figs 11, 14, 27-35). The females differ from the remaining species of the genus by the large epyginal plate and the bulkier anterior lateral spurs in the epigynum (Figs 15, 16, 36-39).

Description. Male (IBSP 36872). Coloration: carapace pale brown, with a dorsal, median, longitudinal yellow band, which extends from the PMA to the posterior border of the carapace. Black thoracic groove, with dark divergent radial lines. Chelicerae reddish brown with two longitudinal black lines. Sternum pale yellow. Legs pale yellow with brown transversal lines; tarsus and metatarsus brown. Abdomen pale yellow with dark grey markings (Fig. 25). Total length 9.40. Carapace 5.30 long, 4.10 wide. Clypeus 0.40 high. Eye diameters and interdistances: AME 0.14, ALE 0.16, PME 0.18, PLE 0.18, AME-AME 0.06, AME-ALE 0.04, PME-PME 0.08, PME-PLE 0.12, ALE-PLE 0.16, AME-AMP 0.06. Leg measurements: I: femur 4.90/patella $1.80 /$ tibia $5.10 /$ metatarsus 4.60/tarsus 2.60/total 19.00; II: 4.70/1.90/4.50/4.00/ 2.00/17.10; III: 4.00/1.50/3.90/4.20/1.80/15.40; IV: 5.30/1.80/ 4.50/6.40/2.30/20.30. Leg formula 4123. Leg spination follows the basic generic pattern. Palp: RTA short, with rounded internal branch and external branch bifid at apex (Figs 29-35); subventral apophysis of RTA large with long hairs (Fig. 27); conductor short, envolving the tip of the embolus; median apophysis sinuous, enlarged and pointed at apex; embolus curved and short, with enlarged base and narrowed tip (Figs 11, 14, 27-28).

Female (IBSP 36871). Coloration as in male, but darker (Fig. 26). Total length 11.87. Carapace 5.30 long, 3.70 wide. Clypeus 0.28 high. Eye diameters and interdistances: AME 0.16, ALE 0.18, PME 0.21, PLE 0.24; AME-AME 0.20, AME-ALE 0.08, PME-PME 0.12, PME-PLE 0.22, ALE-PLE 0.14, AME-AMP 0.06. Leg measurements: I: femur 4.00/patella 1.90/tibia 4.15/metatarsus 3.40/tarsus 1.90/total 15.35; II: 3.90/1.90/3.60/3.20/1.80/14.40; III: 3.50/ 1.50/2.70/3.20/1.60/12.50; IV: 4.50/1.50/3.60/5.00/2.00/16.60. Leg formula as in male. Leg spination follows the basic generic pattern, except tibia I-II p0, r0, III r0-1-1-0, IV r0-1-0, metatarsus I-II v2-2-2, p0, r0; III p1-1-1, IV r0-1-1. Epigynum: median plate triangular or quadrangular, with large depression on the lateral fields (Figs 15, 16, 36-37). Internally with elongate copulatory ducts; spermathecae with median constriction, forming a large base and a small head, fertilization ducts curved (Figs 38, 39).

Variation. Ten males: total length 6.40-9.50; carapace 3.105.40; femur I 4.10-4.90; Ten females: total length 9.00-13.00; carapace 4.80-5.80; femur I 3.50-4.50. The RTA of the male palp is variable, presenting internal branch rounded, oval or rectangular, with or without small distal spur (Figs 27, 29-35). The epigynal plate can be subrectangular with large posterior border (Fig. 36) or hexagonal with narrow posterior border (Fig. 37).

Distribution. Brazil: São Paulo, Rio de Janeiro and Minas Gerais.

Other material examined. BRAZIL, Minas Gerais: Lima Duarte (Parque Estadual do Ibitipoca), 6 females, 6.XI.1993, R. Baptista leg. (MNRJ); 3 males and 2 females, 23.X.1994, R. Baptista leg. (MNRJ 2852); Itamonte, 2 females, 17.X.1990, R. \& A. Baptista leg. (MNRJ). Rio de Janeiro: Teresópolis (Parque Nacional da Serra dos Órgãos, $22^{\circ} 27^{\prime} \mathrm{S} 42^{\circ} 59^{\prime} \mathrm{W}$ ), 42 males and 98 females, 1822.VIII.2001, Equipe Biota leg. (IBSP 36803-36832; 36861; 36862); Teresópolis, 2 females, 03.III.1991, R. \& A. Baptista leg. (MNRJ); Petrópolis (Fazenda Ranchinho Porto da Roça, BR040, Km 78.5), 2 females, 8-15.II.2000, Equipe Biota leg. (IBSP 3685836859); 1 male and 1 female, 11-12.XI.1999, Equipe Biota leg. (IBSP 36867-36868); Resende (Parque Nacional do Itatiaia), 1 male and 1 female, 8-15.VI.2001, Equipe Biota leg. (IBSP 3687136872); 2 females, 9.XI.1990, A. B. Kury leg. (MNRJ); 1 male and 3 females, 13-14.XII.1991, R. \& A. Baptista leg. (MNRJ); 1 male, 14.XII.2004, R.P. Indicatti leg. (IBSP 48096); São Lourenço (Três Picos de Salinas), 1 female, 15.XI.1991, R. \& A. Baptista leg. (MNRJ); Paraty (Estrada Paraty-Cunha), 1 male, 6.I.2005, A.J. Santos leg. (IBSP 48437); São Paulo: São José do Barreiro (Parque

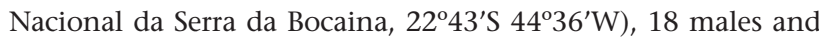
31 females 28.IV-3.V.2002, Equipe Biota leg. (IBSP 36833-36855; 36873-36875); 1 female, 22.X.1960, no collector (MNRJ); Guarulhos (Parque Estadual da Cantareira, 23⒉'15.1"S 46 31'59.7"W), 2 males, 16-22.VII.2001, Equipe Biota leg. (IBSP 36856-36857); Mairiporã (Parque Estadual da Cantareira), 2 female, 15.I.2001, R. Pinto da Rocha et al. leg. (IBSP 36865; MZSP); 2 males and 1 female, 29.X.2000, R. Pinto da Rocha et al. leg. (MZSP); 2 males and 1 female, 29.XI.2000, R. Pinto da Rocha et al. leg. (MZSP); São Paulo (Parque Estadual da Cantareira), 3 males and 2 females, 29.XI.2000, R. Pinto da Rocha et al. leg. (IBSP 36876-36878); 1 female, 24.II.2001, C. Firmo et al. leg. (MZSP); 2 females, 28.II.2001, R. Pinto da Rocha et al. leg. (MZSP); Jundiaí (Parque Estadual da Serra do Japí), 1 male and 2 females, 2728.IV.1991, A.B. Bonaldo leg. (IBSP 6995); 2 males and 6 females, 27-28.IV.1996, A.D. Brescovit leg. (IBSP 7035); 6 males and 50 females, 15-19.VII.2002, Equipe Biota leg. (IBSP 40791-40817); Santana de Parnaíba (Aldeia da Serra), 1 female, 7.VIII.2003, L.B. de Jesus leg. (IBSP 40789); Salesópolis (Reserva Biológica de Boracéia), 1 female, 18-24.V.2001, Equipe Biota leg. (IBSP 40790); 2 females, 19.XI.1991, F.G. Mello leg. (MNRJ); Cotia (Caucaia do Alto), 9 females, III.2003, A. Nogueira \& F.S. Cunha leg. (IBSP).

\section{Itatiaya tacamby sp. nov. Figs $40-43$}

Types. Male holotype from BraziL, Rio de Janeiro: Teresópolis (Parque Nacional da Serra dos Órgãos, 22 $27^{\circ} \mathrm{S}, 42^{\circ} 59^{\prime} \mathrm{W}$ ), 18-22.VIII.2001, Equipe Biota leg., deposited in IBSP 36887.

Revista Brasileira de Zoologia 23 (2): 429-442, junho 2006 

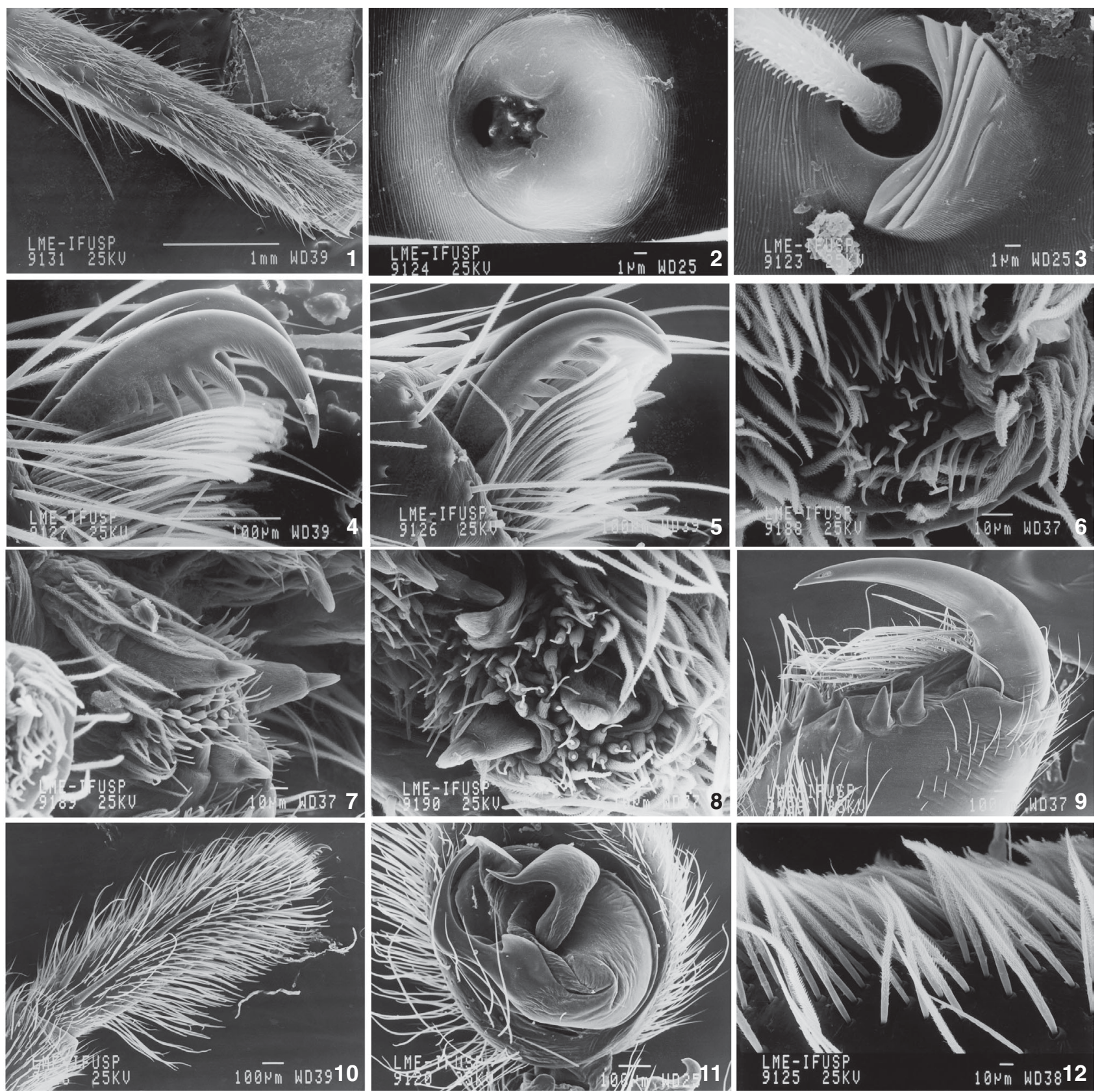

Figures 1-12. (1-5) Itatiaya modesta: (1) tibia I, pairs of spines, lateral view; (2) tarsal organ, female, leg l; (3) trichobothria, male, leg I; (4) claw of tarsus IV, female; (5) claw of tarsus I, male; (6-8) Itatiaya apipema sp. nov.: (6) anterior spinnerets; (7) median spinnerets; (8) posterior spinnerets; (9-12) Itatiaya modesta: (9) female chelicerae, ventral view; (10) female left palp, retrolateral view; (11) male palp, ventral view; (12) male cymbial dorsal scopulae, retrolateral view.

Paratypes: 1 male and 2 females, with the same data as holotype, deposited in IBSP 36885; 36886 and 36888.

Etymology. The specific name originates from a Tupi Indian dialect and means "bifurcate" in reference to the bifurcated median apophysis of the male palp.

Revista Brasileira de Zoologia 23 (2): 429-442, junho 2006
Diagnosis. Itatiaya tacamby sp. n. resembles I. modesta by the morphology of the embolus and conductor, but is distinguished by the morphology of the external branch of RTA and bifid median apophysis in the male palp (Figs 40-41). The females differs from the remaining species of the genus by the 

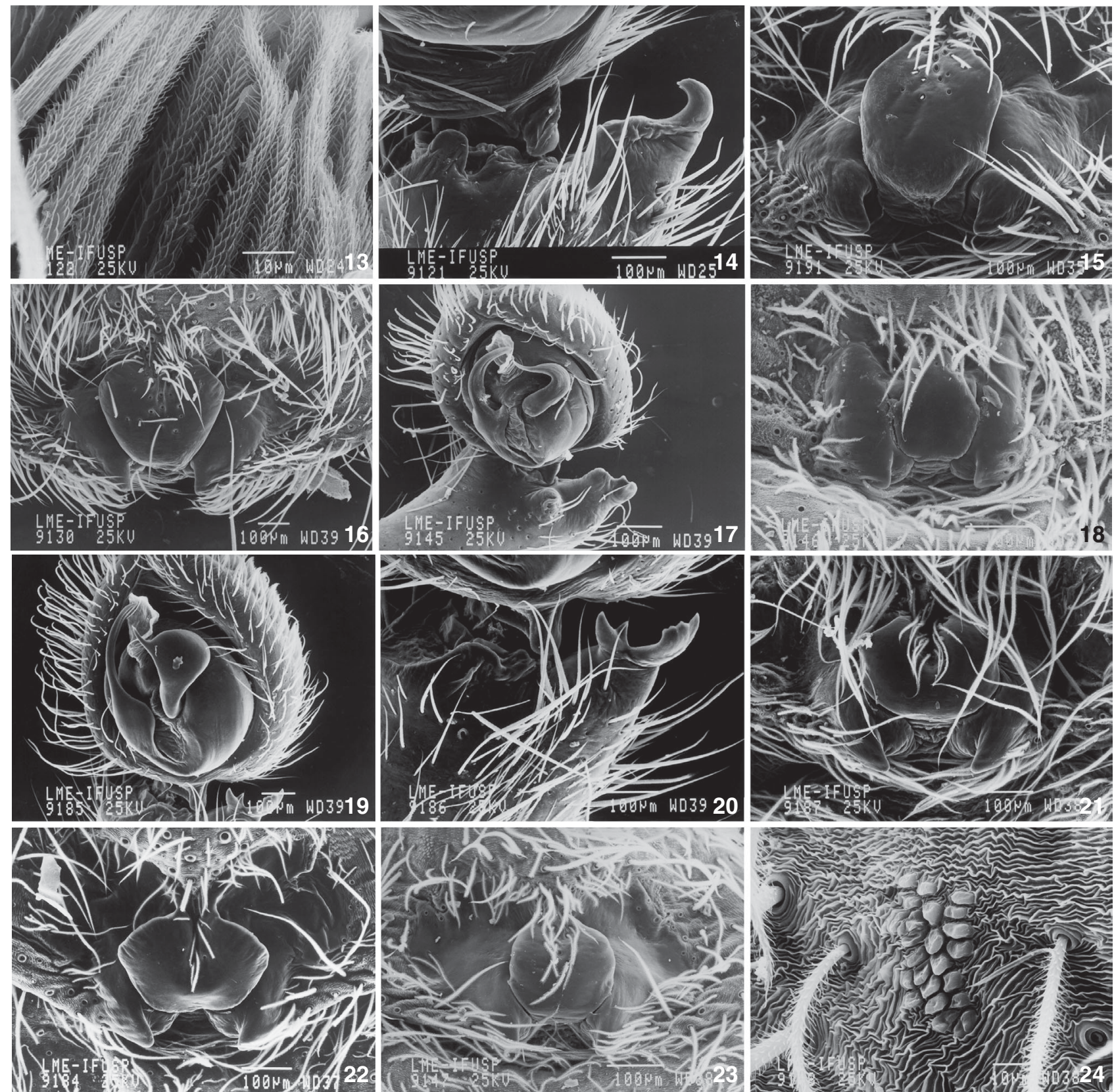

Figures 13-24. (13-16) Itatiaya modesta: (13) male cymbial dorsal scopulae, retrolateral view; (14) RTA of male palp, ventral view; (15) female epigynum, ventral view; (16) female epigynum, variation, ventral view; (17-18) Itatiaya iuba sp. nov.: (17) male palp, ventral view; (18) female epigynum, ventral view; (19-21) Itatiaya apipema sp. nov.: (19) male palp, ventral view; (20) RTA of male palp, ventral view; (21) female epigynum, ventral view; (22) Itatiaya ywyty sp. nov., female epigynum, ventral view; (23-24) Itatiaya pykyyra sp. nov.: (23) female epigynum, ventral view; (24) muscular impression of female epigynum, ventral view.

small median plate and short ventral lateral spurs (Fig. 42) in the epigynum.

Description. Male (holotype). Coloration as in I. modesta. Total length 6.70. Carapace 4.10 long, 3.30 wide. Clypeus 0.10 high. Eye diameters and interdistances: AME 0.16, ALE 0.12, PME 0.17, PLE 0.18, AME-AME 0.06, AME-ALE 0.05, PME-PME 0.04, PME-PLE 0.08, ALE-PLE 0.10, AME-AMP 0.03. Leg measurements: I: femur 4.10/patella 1.40/tibia 4.60/metatarsus 4.20 /

Revista Brasileira de Zoologia 23 (2): 429-442, junho 2006 

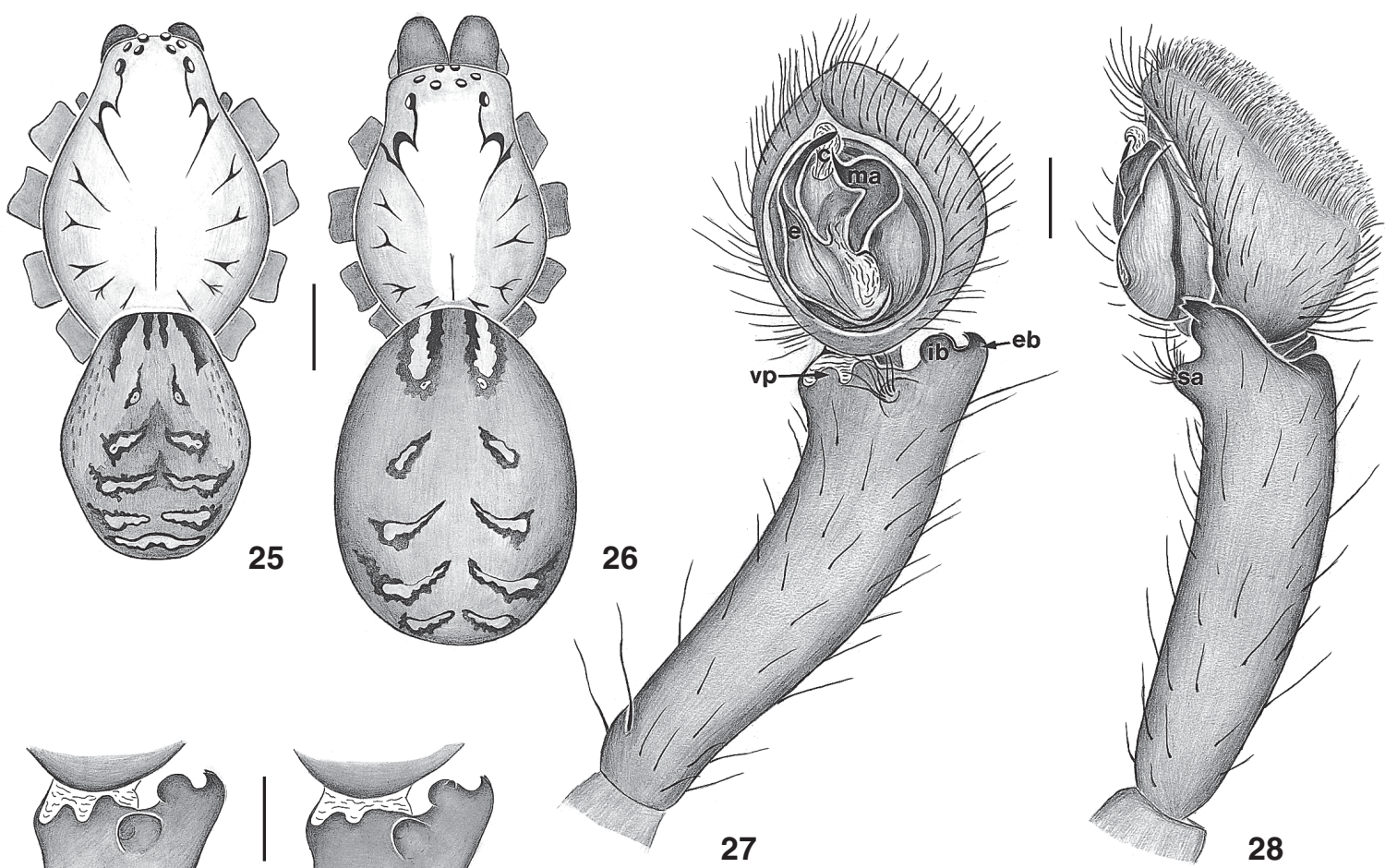

29

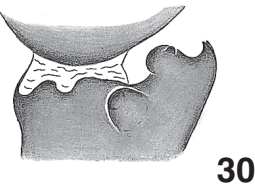

30
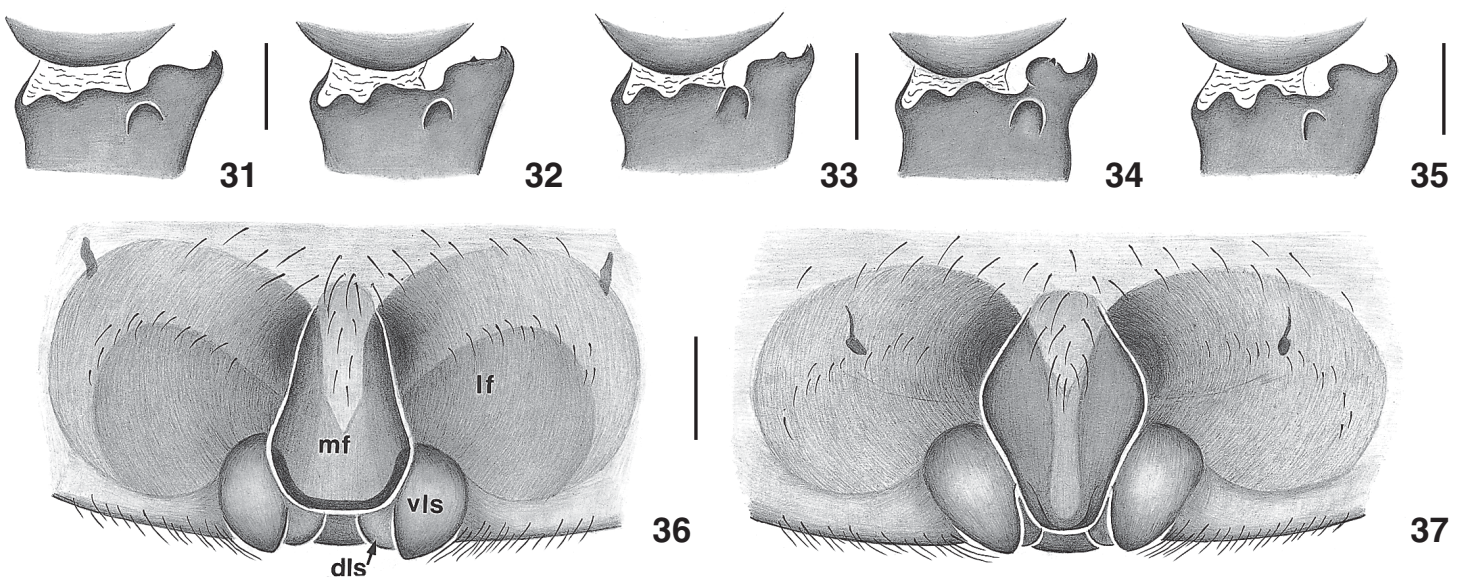

35

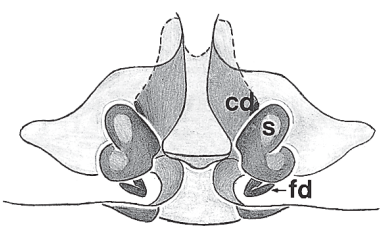

38

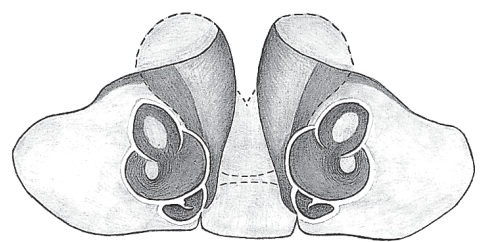

37

Figures 25-39. (25-39) Itatiaya modesta: (25) male body, dorsal view; (26) female body, dorsal view; (27-28) male palp; (27) ventral view: (c) conductor, (e) embolus, (eb) external branch of RTA, (ib) internal branch of RTA, (ma) median apophysis, (vp) ventral projection; (28) retrolateral view: (sa) subventral apophysis; (29-35) RTA of male palp, variation, ventral view: (29) Parque Nacional do Itatiaia, ventral view; (30) Serra do Japí, ventral view; (31) Serra dos Órgãos, ventral view; (32) Petrópolis, Rio de Janeiro, ventral view; (33) Serra da Cantareira, ventral view; (34) Serra da Bocaina, ventral view; (35) Cotia, São Paulo, ventral view; (36-39) female epigyne: (36) ventral view: (dls) dorsal lateral spurs, (If) lateral field, (mf): median field, (vls) ventral lateral spurs; (37) ventral view, variation; (38) dorsal view: (cd) copulatory ducts, (fd) fertilization ducts, (s) spermathecae; (39) dorsal view, variation.

Revista Brasileira de Zoologia 23 (2): 429-442, junho 2006 

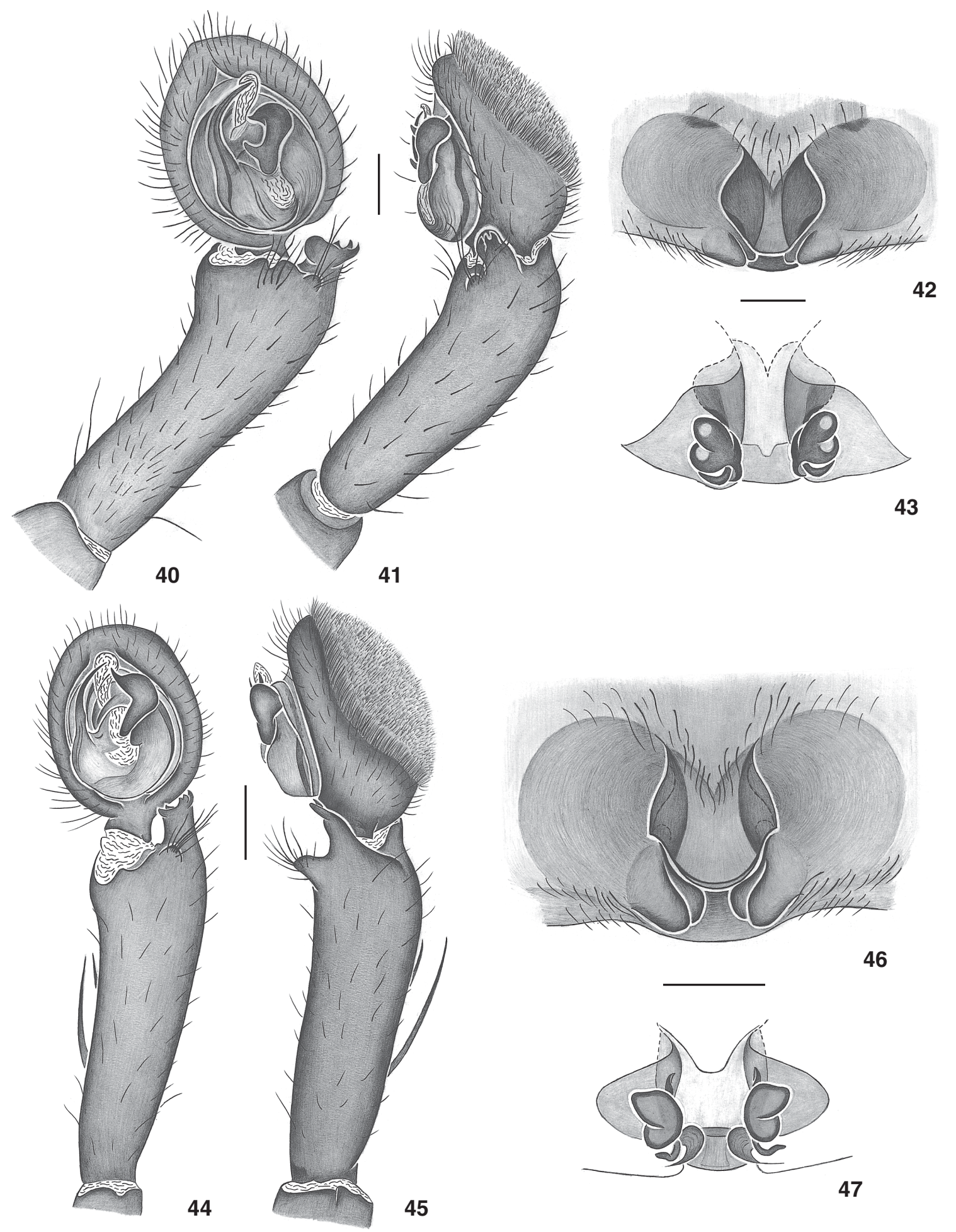

46

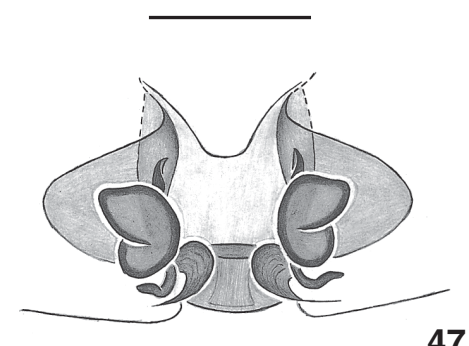

47

Figures 40-47. (40-43) Itatiaya tacamby sp. nov. (40-41) Male palp: (40) ventral view; (41) retrolateral view; (42-43) female epigynum: (42) ventral view; (43) dorsal view; (44-47) Itatiaya apipema sp. nov.: (44-45) male palp: (44) ventral view; (45) retrolateral view; (4647) female epigynum; (46) ventral view; (47) dorsal view. 
tarsus 2.10/total 16.40; II: 3.70/1.40/4.00/3.30/1.50/13.90; III: 3.60/1.20/3.60/3.80/1.80/14.00; IV: 4.60/1.40/4.70/5.90/2.20/ 18.80. Leg formula: 4132 . Leg spination follows the basic generic pattern, except: tibia I r1-1-1; metatarsus I v2-2-2, p0-11-0; II v2-2-2, p1-1-1, r1-1-1; III p1-1-1; IV v2-2-2-1v, p1-1-1. Palp: tibia distally large, RTA with complex external branch, presenting three projections, and with a small subventral apophysis of RTA; median apophysis bifid; conductor envolving the tip of the embolus; embolus curved with enlarged base and narrowed tip (Figs 40, 41).

Female (IBSP 36885). Coloration darker than males. Total length 9.10. Carapace 4.10 long, 3.10 wide. Clypeus 0.18 high. Eye diameters and interdistances: AME 0.16, ALE 0.16, PME 0.20, PLE 0.21, AME-AME 0.10, AME-ALE 0.08, PME-PME 0.09, PME-PLE 0.14, ALE-PLE 0.16, AME-AMP 0.06. Leg measurements: I: femur 3.40/patella 1.40/tibia 3.40/metatarsus $2.70 /$ tarsus 1.50/total length 12.40; II: 3.20/1.20/3.10/2.50/1.10/ 11.10; III: 3.00/1.20/2.60/2.40/1.20/10.40; IV: 3.80/1.30/3.50/ 4.40/1.60/14.60. Leg formula: 4123. Leg spination follows the basic generic pattern, except: tibia I-II p0, r0; metatarsus I-II v2-2-2, p0, r0, III p1-1-1, r2-1-0, IV v2-2-2-1v, r1-1-1-1. Epigynum: triangular and small median plate, with large depressions of the lateral fields and small lateral spurs (Fig. 42). Internally, spermathecae with short copulatory ducts and curved fertilization ducts (Fig. 43).

Variation. Two males: total length 6.70-6.90; carapace 3.70-3.90; femur I 3.90-4.90; two females: total length 6.2012.25; carapace 3.10-6.00; femur I 2.35-5.30.

Distribution. Brazil: Rio de Janeiro.

Other material examined: BrAzIL. Rio de Janeiro: Teresópolis (Parque Nacional da Serra dos Órgãos, 22 $2^{\circ} 27^{\prime}$ S, 42 ${ }^{\circ} 59^{\prime} \mathrm{W}$ ), 1 male, 18-22.VIII.2001, Equipe Biota leg. (IBSP 40543).

\section{Itatiaya apipema sp. nov. Figs 6-8, 19-21, 44-47}

Types. Male holotype from Brazil, São Paulo: Paranapiacaba (Estação Biológica do Alto da Serra), XI.1990, R. \& A. Baptista leg., deposited in IBSP 40819. Paratypes: 1 female, with same locality as holotype, 27.VII.2000, F.S. Cunha leg. (IBSP 23979); 1 male and 1 female, from Brasil, Rio de Janeiro: São Lourenço (Três Picos de Salinas), 15.XI.1991, R. Baptista leg. (IBSP 40820, 40821).

Etymology. The specific name originates from the Tupi Indian dialect and means "the top of a mountain", and refers to the collection site.

Diagnosis. Itatiaya apipema sp. nov. resembles I. modesta by the structures of the palpal tegulum, but is distinguished by the slender embolus and by complex RTA with four projections (Figs 19, 20, 44, 45) in the male palp and by the semicircular posterior border of the median plate and large ventral and dorsal lateral spurs (Figs 21, 46) in the female epigynum.

Description. Male (holotype). Coloration as in I. modesta. Total length 6.10. Carapace 3.10 long, 2.40 wide. Clypeus 0.08 high. Eye diameters and interdistances: AME 0.14, ALE 0.12, PME 0.18, PLE 0.18, AME-AME 0.02, AME-ALE 0.01, PME-PME 0.06, PME-PLE 0.05, ALE-PLE 0.04, AME-AMP 0.03. Leg measurements: I: femur 3.10/patella 1.10/tibia 3.63/metatarsus 3.15/ tarsus 1.45/total 12.43; II: 2.95/1.10/3.15/2.80/1.20/11.20; III: 2.55/1.20/3.25/2.95/1.15/11.10; IV: 3.05/1.00/3.55/4.30/1.55/ 13.45. Leg formula: 4123 . Leg spination follows the basic generic pattern, except: tibia I-II p0, r0, III r0-1-1-0, IV r11-1, metatarsus I-II v2-2-2, p0, r0, III p1-1-2, r1-1-2, IV v2-2-2, p11-2, r1-1-1-2. Palp: straight tibia, complex RTA with four projections, rounded subventral apophysis of RTA, covered by long hairs; ventral projection of tibia very short; conductor large and hyaline, envolving the tip of the embolus; median apophysis medialy enlarged, slender embolus (Figs 19-20, 44-45).

Female (IBSP 23979). Coloration: as in male. Total length 8.15. Carapace 3.50 long, 2.60 wide. Cypeus 0.12 high. Eye diameters and interdistances: AME 0.14, ALE 0.16, PME 0.18, PLE 0.14, AME-AME 0.06, AME-ALE 0.05, PME-PME 0.08, PMEPLE 0.10, ALE-PLE 0.13, AME-AMP 0.04. Leg measurements: I: femur 2.60/patella 1.10 /tibia 2.85 /metatarsus $2.05 /$ tarsus $0.95 /$ total 9.55; II: 2.65/0.95/2.40/2.10/0.85/8.95; III: 2.40/0.90/2.08/ 2.30/0.90/8.58; IV: 2.90/0.95/2.55/3.30/1.30/11.00. Leg formula: 4123. Leg spination follows the basic generic pattern, except: tibia I-II p0, r0, III r0-1-1-0; metatarsus I-II v2-2-2, p0, r0; III p1-1-2, r1-1-2; IV v2-2-2-1v, p1-1-2, r1-1-2. Epigynum: subquadrangular median plate with a semicircular posterior border, large depression of the lateral field and large ventral and dorsal lateral spurs (Figs 21, 46). Internally with spermathecae with median constriction, forming a small base and large head; copulatory ducts elongated, fertilization ducts long, sinuous and slender (Fig. 47).

Variation. Six males: total length 5.50-7.10; carapace 2.903.50; femur I 2.90-3.25; seven females: total length 6.30-8.20; carapace 3.20-3.45; femur I 2.10-2.75.

Distribution. Brazil: Rio de Janeiro and São Paulo.

Other material examined. BRAzIL, Rio de Janeiro: São Lourenço (Três Picos de Salinas), 3 males and 5 females, 15.XI.1991, R. \& A. Baptista leg. (MNRJ). São Paulo: Paranapiacaba, Estação Biológica do Alto da Serra, 3 males and 3 females, XI.1990, R. $\&$ A. Baptista leg. (MNRJ 11904).

\section{Itatiaya iuba sp. nov. Figs 17, 18, 48-51}

Types. Male holotype and female paratype from BRASIL, São Paulo: Mogi das Cruzes (Parque Natural Municipal da Serra do Itapety), 06.VII.2000, S.S. Nagaki leg., deposited in IBSP 36890 and 40818. Paratypes: 1 male, São Paulo: Mairiporã (Parque Estadual da Serra da Cantareira, Pinheirinho), 29.IV.2001, C.L. Firmo et al. leg. (IBSP 36894); 1 female, same locality and collector, 26.VII.2001 (IBSP 36893). 1 male and 1 female, same locality, 13.VIII.2000, R. Pinto da Rocha et al. leg., deposited in MZSP 25691 and 25692.

Etymology. The specific name originates from the Latin

Revista Brasileira de Zoologia 23 (2): 429-442, junho 2006 

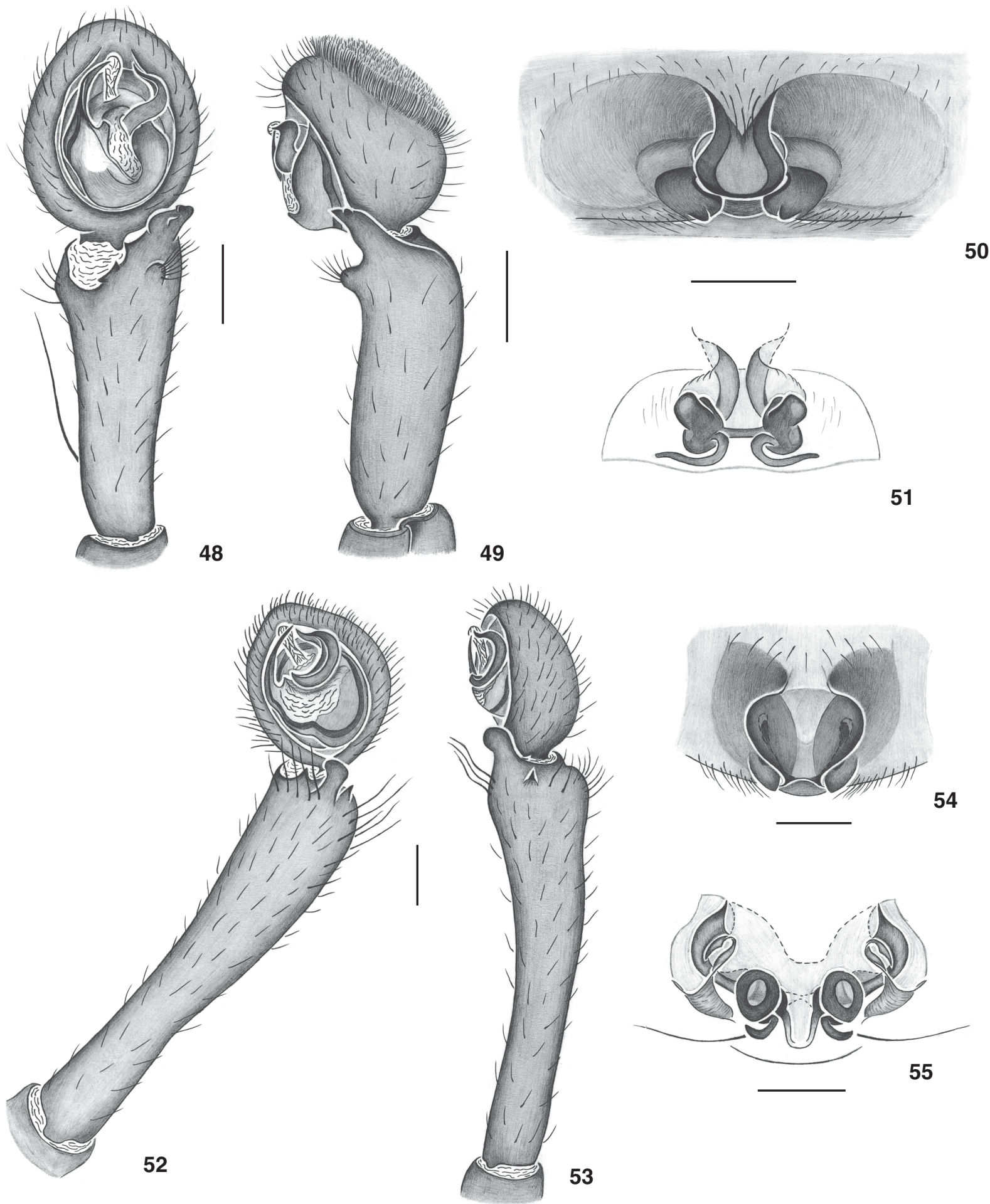

51 50

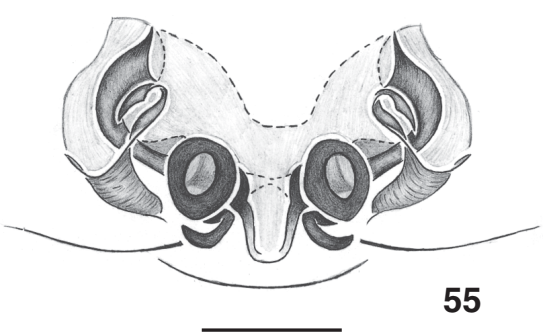

Figures 48-55. (48-51) Itatiaya iuba sp. nov. (48-49) Male palp: (48) ventral view; (49) retrolateral view; (50-51) female epigyne: (50) ventral view; (51) dorsal view; (52-55) Itatiaya pucupucu sp. nov.: (52-53) male palp: (52) ventral view; (53) retrolateral view; (54-55) female epigynum: (54) ventral view; (55) dorsal view. 
and means tuft, crest or wig, and refers to the cymbium scopulae on male palp.

Diagnosis. Itatiaya iuba sp. nov. differs from the remaining species of the genus by the slender and sinuous median apophysis and by the morphology of the extenal branch of the RTA (Figs 17, 48, 49) in the male palp and by the semicircular median plate with a small ventral lateral spurs (Figs 18,50) in the female epigynum.

Description. Male (MZSP 25691). Coloration as in $I$. modesta. Total length 4.30. Carapace $2.30 \mathrm{long}, 1.90$ wide. Clypeus 0.03 high. Eye diameters and interdistances: AME 0.08; ALE 0.10; PME 0.16; PLE 0.18, AME-AME 0.06, AME-ALE 0.04, PME-PME 0.05, PME-PLE 0.045, ALE-PLE 0.09, AME-AMP 0.05. Leg measurements: I: femur $1.95 /$ patella $0.75 /$ tibia $2.05 /$ metatarsus 1.70/tarsus 0.95/total 7.40; II: 1.77/0.75/1.70/1.55/0.75/ 6.52; III: 1.65/0.70/1.45/1.70/0.75/6.25; IV: 2.10/0.65/1.80/ 2.40/1.02/7.97. Leg formula: 4123. Leg spination follows the basic generic pattern, except: tibia IV r1-1-1; metatarsus I v2-22, p0-1-1-0, r0-1-1-0; II v2-2-2, p1-1-1, r1-1-1; III p1-1-2, r1-12, IV v2-2-2, p1-1-2, r1-2-2. Palp: short tibia, round internal branch of RTA and complex external branch of RTA with two projections, slender and sinuous median apophysis, short and rounded subventral apophysis of RTA, covered by long distal hairs; ventral projection of tibia short and truncated; embolus short, with large base (Figs 17, 48, 49).

Female (MZSP 25692). Coloration as in male. Total length 4.16. Carapace 2.04 long, 1.92 wide. Clypeus 0.02 high. Eye diameters and interdistances: AME 0.10; ALE 0.11; PME 0.14; PLE 0.16, AME-AME 0.05, AME-ALE 0.03, PME-PME 0.04, PMEPLE 0.03, ALE-PLE 0.05, AME-AMP 0.03. Leg measurements: I: femur 1.86 /patella $0.76 /$ tibia $2.04 /$ metatarsus 1.68 /tarsus 0.88 / total 7.22; II: 1.76/0.68/1.66/1.48/0.60/6.18: III: 1.64/0.56/1.16/ 1.68/0.88/5.92; IV: 2.04/0.60/1.80/2.44/0.70/7.58. Leg formula: 4123. Leg spination follows the basic generic pattern, except: tibia III r0-1-1-0; metatarsus I v2-2-2, r0-1-1; II v2-2-2, p1-1-1, r1-1-1; III p1-1-2, r1-1-2, IV v2-2-2, p1-1-1, r0-2-2. Epigynum: semicircular median plate invaginated anteriorly, small ventral and dorsal lateral spurs, depression of the lateral field deeply excaveted (Figs 18, 50). Internally, spermathecae with median constriction, forming large base and subquatrate head; copulatory ducts elongated; fertilization ducts long and slender (Fig. 51).

Variation. Ten males: total length 3.95-4.80; carapace 2.25-2.50; femur I 1.65-2.05; ten females: total length 5.206.70; carapace 2.60-2.90; femur I 1.75-1.95.

Distribution. Brazil: São Paulo.

Other material examined. BRAziL, São Paulo: Mogi das Cruzes (Parque Natural Municipal da Serra do Itapety), 1 male and 6 females, 6.VII.2000, S.S. Nagaki leg. (IBSP 36890-36891); 3 males and 9 females, 13-19.X.2003, Equipe Biota leg. (IBSP $47902-$ 47909); São Paulo (Parque Estadual da Cantareira), 7 males and 2 females, 13.VIII.2000 (IBSP 36892; MZSP); 3 females, 29.X.2000 (MZSP); 1 female, 29.XI.2000 (MZSP); 1 male and 1 female,
21.XII.2000, all collected by R. Pinto da Rocha et al. leg. (MZSP); 1 female, 22-27.IV.2001 (MZSP); 1 female, X.2000 (IBSP 36895); 1 male, 24.II.2001 (IBSP 36896); 2 males and 4 females, 2000 (MZSP); 1 female, 7.IV.2000 (MZSP); 1 male, 17.XI.2000 (IBSP 36897); 3 males and 2 females, 2000 (MZSP); 1 female, VIII. 2000 (IBSP 36898); 2 males and 1 female, 27.IV.2001, all collected by C.L. Firmo et al. leg. (MZSP); Mairiporã, Parque Estadual da Cantareira, 1 female, 27.IV.2001, C.L. Firmo et al. leg. (MZSP); 1 male, 29.IV.2001, C.L. Firmo et al. leg. (MZSP).

\section{Itatiaya рuсupucu sp. nov.}

\section{Figs $52-55$}

Types. Male holotype and two female paratypes from Brazil, Minas Gerais: Alto Caparaó (Parque Nacional da Serra do Caparaó), 1-7.V.2002, Equipe Biota leg. deposited in IBSP 40672, 40673, 40674, respectively.

Etymology. The specific name originates from the Tupi Indian dialect and means "very long", and refers to the very long tibia of the male palp.

Diagnosis. Itatiaya puсириси sp. nov. is easily distinguished from other species of the genus by the very long tibia, RTA reduced to a spiniform projection, elongated subventral apophysis of RTA, half-moon shape median apophysis and cymbium without dorsal scopulae (Figs 52,53) in the male palp. The females can be distinguished by the reduced depressions of the lateral fields and cordiform median plate (Fig. 54) in the epigynum.

Description. Male (holotype). Coloration as in I. modesta. Total length 6.20. Carapace 3.30 long, 2.60 wide. Clypeus 0.09 high. Eye diameters and interdistances: AME 0.08, ALE 0.11, PME 0.16, PLE 0.14, AME-AME 0.08, AME-ALE 0.03, PME-PME 0.06, PME-PLE 0.08, ALE-PLE 0.11, AME-AMP 0.07. Leg measurements: I: femur 2.80/patella 1.05/tibia 2.65/metatarsus 2.30/ tarsus 1.30/total 10.10; II: 2.20/1.00/2.40/2.30/1.20/9.10; III: 2.30/0.80/1.80/2.50/1.00/8.40; IV: 3.30/1.00/2.60/3.60/1.40/ 11.90. Leg formula: 4123. Leg spination follows the basic generic pattern, except: tibia III r0-1-1-0; metatarsus I v2-2-1v, p1-1-2, r0-1-1, II v2-0-1v, p1-1-2, r0-1-1, IIII v2-2-2, p1-1-1, r02-2, IV v2-2-2-1v, p1-1-2, r1-1-2. Palp: long tibia, almost four times longer than cymbium, cymbium without scopulae, RTA reduced to a spiniform projection, elongated subventral apophysis of RTA, half-moon shape median apophysis, short and conical ventral projection, slender embolus with enlarged base and long conductor (Figs 52, 53).

Female (Paratype IBSP 40673). Coloration as in male. Total length 7.10. Carapace 3.40 long, 2.50 wide. Clypeus 0.14 high. Eye diameters and interdistances: AME 0.08, ALE 0.11, PME 0.16, PLE 0.14, AME-AME 0.08, AME-ALE 0.04, PME-PME 0.05, PME-PLE 0.09, ALE-PLE 0.06, AME-AMP 0.04. Leg measurements: I: femur 2.15/patella 1.15/tibia 2.10/metatarsus 1.75/tarsus 0.95/ total 8.10; II: 2.15/1.00/2.00/1.75/0.90/7.80; III: 1.90/0.80/1.45/ 1.45/0.85/6.45; IV: 2.60/0.90/2.05/2.70/1.15/9.40. Leg formula: 4123. Leg spination follows the basic generic pattern, except: tibia I-II p0, r0, III r0-1-1-0; metatarsus I v2-2-2, p0, r0, II p0, r0, 
III p0-1-2, r1-1-2, IV v2-2-2-1v, p1-1-1, r1-1-2. Epigynum: median plate with curved posterior border, reduced depressions of the lateral fields, short ventral lateral spurs and inconspicuous dorsal lateral spurs (Fig. 54). Internally, spermathecae with reduced base and large and rounded head, elongated and curved copulatory ducts, curved fertilization ducts (Fig. 55).

Variation. Four females: total length 7.70-10.80; carapace 3.70-4.90; femur I 2.30-3.20.

Distribution. Known only from the type locality.

Other material examined. BraziL, Minas Gerais: Alto Caparaó (Parque Nacional da Serra do Caparaó), 2 females and 1 immature, 1-7.V.2002, Equipe Biota leg. (IBSP 36889)

\section{Itatiaya pykyyra sp. nov. \\ Figs 23, 24, 56, 57}

Types. Female holotype from BraziL, Minas Gerais: Alto Caparaó (Parque Nacional da Serra do Caparaó), 1-7.V.2002, Equipe Biota leg., deposited in IBSP 40544. Paratypes: 2 females, with same data as holotype deposited in IBSP 36925, 36928 and 2 females with same data as holotype deposited in MZSP 25693.

Etymology. The specific name originates from a Tupi Indian dialect and means "younger sister", and refers to smaller species of Itatiaya collected in Parque Nacional da Serra do Caparaó.

Diagnosis. Itatiaya pykyyra sp. nov. can be distinguished from other species of the genus by the rectangular median plate, short ventral lateral spurs and conical dorsal lateral spurs (Figs $23,56)$ in the epigynum.

Description. Male unknown.

Female (holotype). Coloration as in I. modesta. Total length 6.90. Carapace 2.90 long, 2.40 wide. Clypeus 0.10 high. Eye diameters and interdistances: AME 0.14, ALE 0.14, PME 0.16, PLE 0.16, AME-AME 0.05, AME-ALE 0.04, PME-PME 0.10, PME-PLE 0.12, ALE-PLE 0.10, AME-PME 0.04. Leg measurements: I: femur 2.35/patella 0.90/tibia 2.45/metatarsus $2.00 /$ tarsus 1.10/total 8.80; II: 2.40/0.95/2.15/1.80/1.00/8.30; III: 2.15/0.75/1.83/1.95/1.00/7.68; IV: 2.70/0.90/2.55/3.20/1.45/ 10.80. Leg formula: 4123 . Leg spination follows the basic generic pattern, except: tibia I-II p0, r0, III r0-1-1-0; metatarsus III v2-2-2, p0, r0; III p1-1-2, r1-1-2; IV p1-1-2, r1-1-0. Epigynum: rectangular median plate with rounded and chitinous posterior border, short ventral lateral spurs, conical dorsal lateral spurs, depression of the lateral fields deeply excaveted (Fig. 56). Internally with elongated copulatory ducts, spermathecae with median constriction, forming a base and head of almost the same size, curved fertilization ducts (Fig. 57).

Variantion. Ten females: total length 6.10-7.20; carapace 2.80-3.20; femur I 2.20-2.40.

Distribution. Known only from the type locality.

Other material examined. BraziL, Minas Gerais: Alto Caparaó (Parque Nacional da Serra do Caparaó), 26 females, 17.V.2002, Equipe Biota leg. (IBSP 36927; 36929-36933).

\section{Itatiaya tubixaba sp. nov.}

Figs 58, 59

Types. Female holotype and female paratype from Brazil, Minas Gerais: Alto Caparaó (Parque Nacional da Serra do Caparaó), 1-7.V.2002, Equipe Biota leg., deposited in IBSP 36869 and 36870 .

Etymology. The specific name originates from a Tupi Indian dialect and means "bigger", and refers to the largest Itatiaya species collected at the Parque Nacional da Serra do Caparaó.

Diagnosis. Itatiaya tubixaba sp. nov. is distinguished from remaining species by the sulcated anterior border of median plate, voluminous ventral lateral spurs and small depression of the lateral fields (Fig. 58) in the epigynum.

Description. Male unknown.

Female (Holotype). Coloration as in I. modesta. Total length 12.13. Carapace 5.50 long, 4.30 wide. Clypeus 2.25 high. Eye diameters and interdistances: AME 0.18, ALE 0.19, PME 0.21, PLE 0.22, AME-AME 0.10, AME-ALE 0.10, PME-PME 0.17, PMEPLE 0.20, ALE-PLE 0.15, AME-AMP 0.15. Leg measurements: I: femur 5.10/patella 2.00/tibia 5.30/metatarsus $4.00 /$ tarsus 1.80 / total 18.20; II: 5.05/2.00/4.85/3.90/1.70/17.50; III: 4.30/1.80/ 3.70/3.70/1.50/15.00; IV: 5.50/1.90/5.30/6.30/2.10/21.10. Leg formula: 4123. Leg spination follows the basic generic pattern, except: tibia I-II p0, r0, III p0-1-0, r1-0-1, IV r1-0-1; metatarsus I-II v2-2-2, p0, r0, III p0-1-1-0, r0-1-1-0, IV p1-1-2, r0-1-1-0. Epigynum: sulcated anterior border of median plate, voluminous ventral lateral spurs, small dorsal lateral spurs and small depression of the lateral fields (Fig. 58). Internally with elongated copulatory ducts, spermathecae with median constriction, forming a small base and large and oval head, short fertilization ducts (Fig. 59).

Distribution. Brazil: Minas Gerais.

Other material examined. None.

\section{Itatiaya ywyty sp. nov.}

\section{Figs 22, 60, 61}

Types. Female holotype from Brazil, Rio de Janeiro: Teresópolis, 20-22.IV.1995, R. Baptista \& M.I. Landim leg., deposited in IBSP 40882. Paratypes: 2 females, with same data as holotype deposited in IBSP 40823 and 40824.

Etymology. The specific name originates from the Oiampí Indian dialect and means "mountain" and refers to the collection site.

Diagnosis. Itatiaya ywyty sp. nov. can be distinguished from remaining species of the genus by the large median plate and short ventral lateral spurs (Figs 22,60) in the epigynum.

Description. Male unknown.

Female (holotype). Coloration as in I. modesta. Total length 8.00. Carapace 3.70 long, 3.00 wide. Clypeus 0.22 high. Eye diameters and interdistances: AME 0.16, ALE 0.16, PME 0.19, PLE 0.20, AME-AME 0.12, AME-ALE 0.06, PME-PME 0.07, PME-PLE 0.16, ALE-PLE 0.17, AME-PME 0.13. Leg measurements: I: femur 3.05/patella 1.25/tibia 3.15/metatarsus 2.45 / tarsus 1.05/total 10.95; II: 2.90/1.25/2.85/2.30/1.00/10.30; III: 

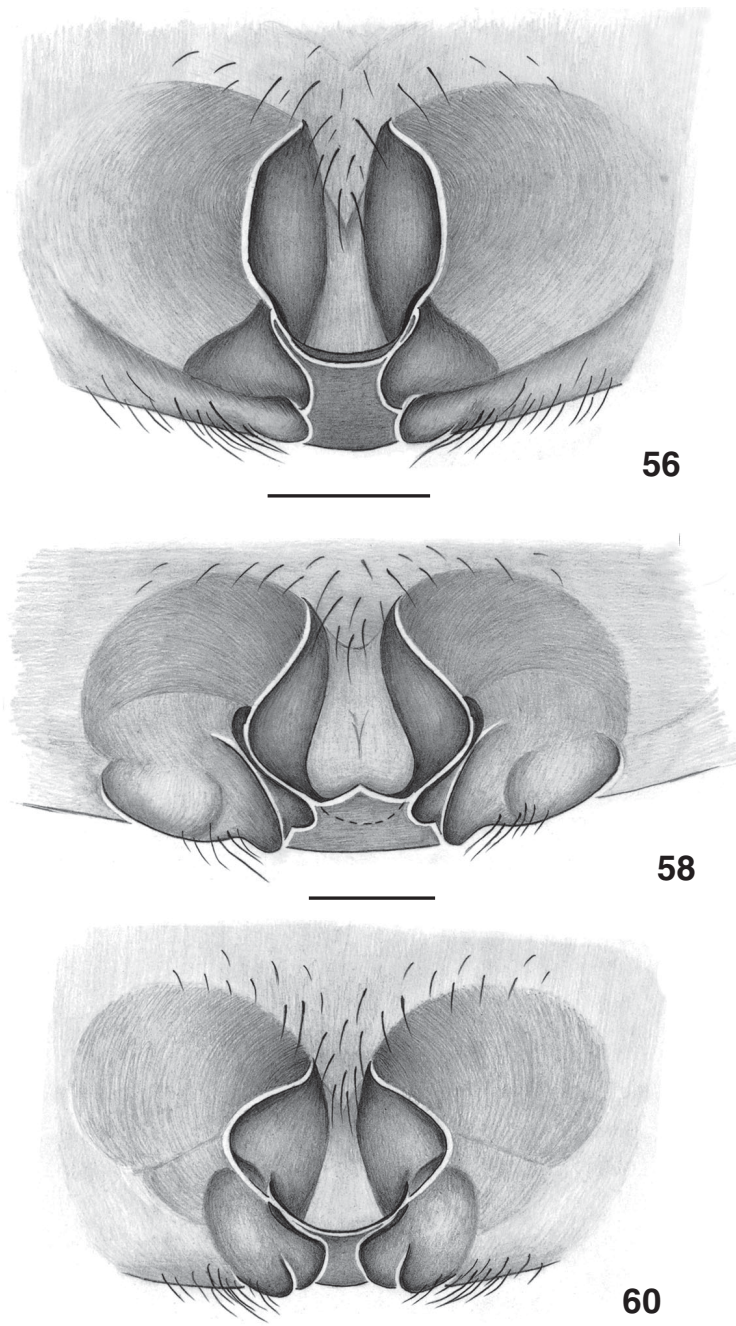

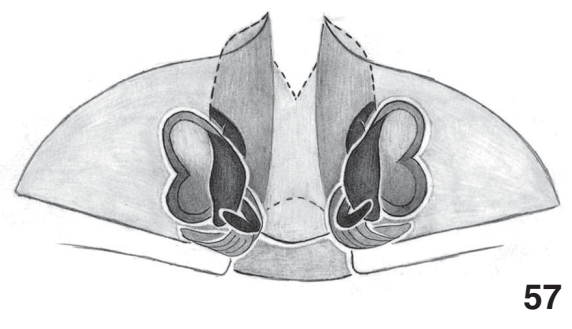

57

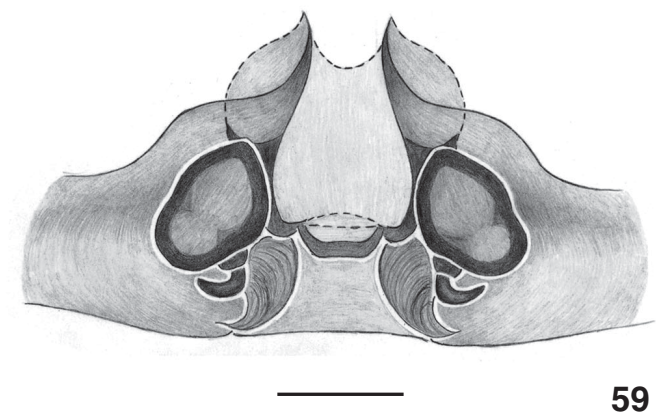

59

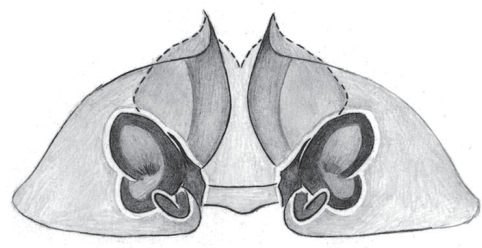

61

Figures 56-61. (56-57) Itatiaya pykyyra sp. nov.: (56) female epigynum, ventral view; (57) dorsal view; (58-59) Itatiaya tubixaba sp. nov.: (58) female epigynum, ventral view; (59) dorsal view; (60-61) Itatiaya ywyty sp. nov.: (60) female epigynum, ventral view; (61) dorsal view.

2.53/0.95/2.35/2.55/1.05/9.43; IV: 3.50/1.10/3.25/4.05/1.45/ 13.35. Leg formula: 4123 . Leg spination follows the basic generic pattern, except: tibia I -II p0, r0, III r0-1-1-0, metatarsus I-II v2-2-2, p0, r0, III-IV p1-1-2, r1-1-2. Epigynum: large median plate with rounded posterior border, depression of the lateral fields deeply excavated, short ventral and dorsal lateral spurs (Fig. 60). Internally with elongated copulatory ducts, spermathecae with median constriction, forming short base and elongated head, curved fertilization ducts (Fig. 61).

Variation. Five females: total length 6.90-8.30; carapace 3.30-3.80; femur I 2.60-3.20.

Distribution. Brazil: Rio de Janeiro.

Other material examined. BRAzIL, Rio de Janeiro: Teresópolis, 7 females, 20-22.IV.1995, R. Baptista \& M.I. Landim leg. (MNRJ 2824).

Revista Brasileira de Zoologia 23 (2): 429-442, junho 2006

\section{GEOGRAPHICAL DISTRIBUITION}

Species of the genus Itatiaya show a distribution pattern related to altitude forested areas. All the eight species were collected in restricted mountainous regions in southeastern Brazil, at least $500 \mathrm{~m}$ above sea level. With the exception of $I$. apipema sp. nov., I. iuba sp. nov. and I. modesta, all species seem to present an endemic distribution pattern.

Itatiaya apipema sp. nov. was collected at Serra do Paranapiacaba, on the coast of São Paulo state, and Serra dos Órgãos, in Rio de Janeiro state. In Serra dos Órgãos this species is sympatric with I. modesta. Itatiaya iuba sp. nov. was collected in two different, but closer, sites: Serra da Cantareira and Serra do Itapety. Itatiaya modesta is the most widely distributed species, with records from several mountains in the states of São 


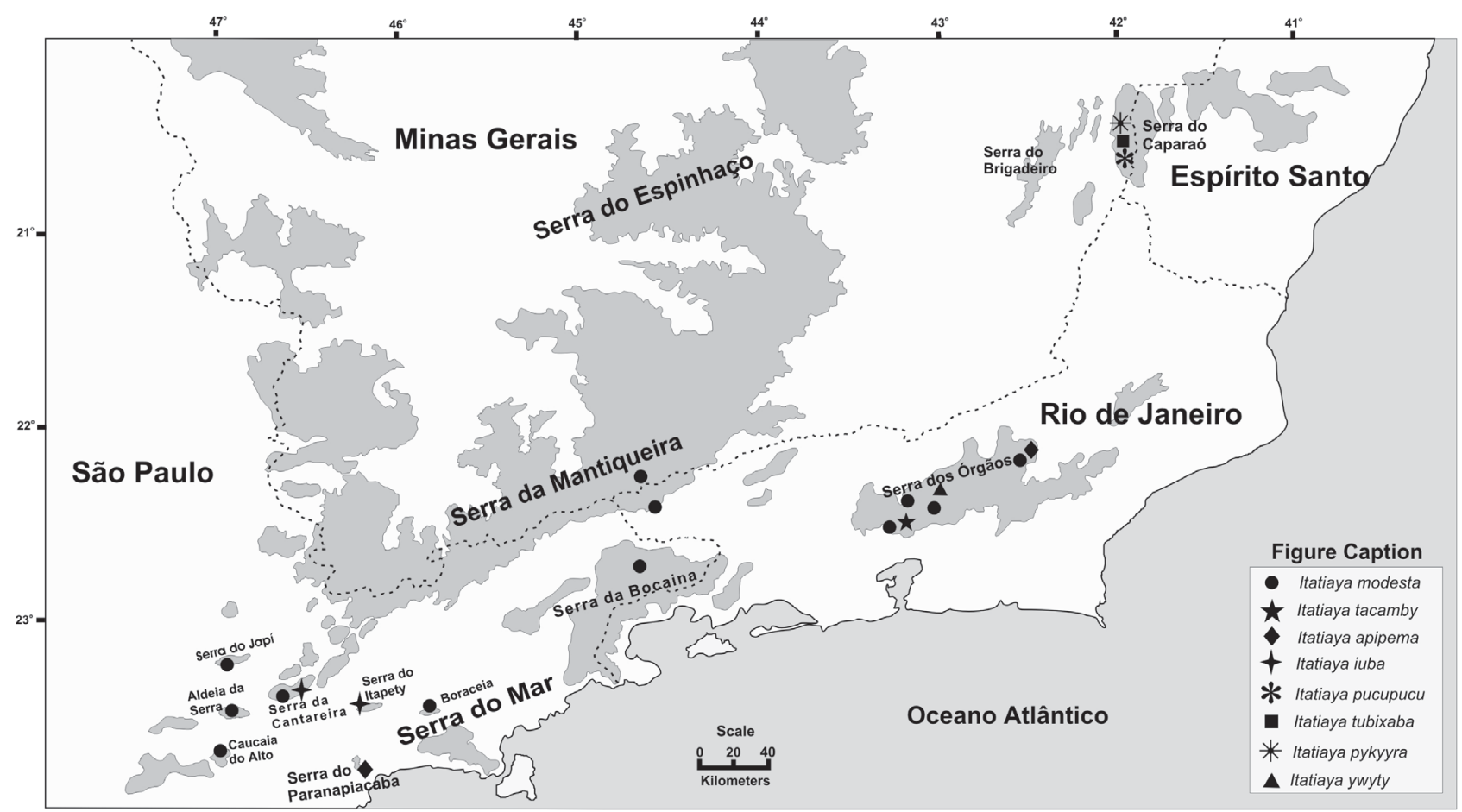

Figures 62. Colection sites and distribution pattern of Itatiaya in mountanious region in southest of Brazil.

Paulo, Rio de Janeiro and Minas Gerais.

The other five species, I. tacamby sp. nov., I. pucupucu sp. nov., I pykyyra sp. nov., I. tubixaba sp. nov. and I. ywyty sp. nov. presented an endemic distribution and are restricted to a single area. At Serra do Caparaó, a case of sympatry was observed, with $I$. puсириси sp. nov., I. pykyyra sp. nov. and $I$. tubixaba sp. nov. being collected in the same site, but at different altitudes (no data available). Itatiaya tacamby sp. nov. and I. ywyty sp. nov. occured only in Serra dos Órgãos and are sympatric with $I$. modesta, but the species were collected at different altitudes.

In the Neotropical region areas of endemism were proposed by Platnick (1976), for spiders of the subfamily Laroniinae (Gnaphosidae), and by BERTANI (2001), for the subfamily Theraphosinae (Theraphosidae). Within Arachnida, the groups that present the best knowledge on distribution patterns are scorpions and harvestmen. Biogeographic notes were presented for scorpions from the Brazilian "matas de brejo" and Atlantic Forest by LOURENÇO (1986) and for harvestmen from southeastern Brazil by PINTO-DA-Rocha (2002) and Tourinho-Davis (2004). The most interesting papers on the biogeography of montane spiders dealt with spiders from African and Malagasy forests (GRISWOLD 1991, GRISWOLD \& LEDFORD 2001).

The endemic areas presented by PINTO-DA-Rocha (2002) seem to be applicable for the distribution of the species of Itatiaya, enabling a preliminary discussion until new samples and new species are discovered in other mountainous areas from southeastern Brazil.

Among the endemic areas proposed by PINTO-DA-RochA (2002: 366, fig. 1), I. modesta occurred in the Serra do Mar, limited to the east by the ocean, to the west by the subtropical forest of the São Paulo Plateau, to the South by the Ribeira de Iguape river valley and to the North by the Ubatuba county and neighboring areas of the Serra da Bocaina, and Serra da Mantiqueira, the type locality. Nevertheless, up north this species is only present in the Serra dos Órgãos. Itatiaya apipema sp. nov. occured in Serra do Mar and Serra dos Órgãos. Itatiaya tacamby sp. nov. and $I$. ywyty sp. nov. occured only in Serra dos Órgãos and are sympatric with I. modesta (Fig. 62), although living at different altitudes. Itatiaya iuba sp. nov. is restrict to Serra do Mar, ocurring in the Serra da Cantareira and in the Serra do Itapety where it is sympatric with $I$. modesta, but smaller in size. The remaining species, I. puсириси sp. nov., I. pikyyra sp. nov. and I. tubixaba sp. nov., were collected together only in the Serra do Caparaó (Fig. 62). However, the collection site is quite far from the border of Serra do Espinhaço, beside the Serra do Brigadeiro, where no records of Itatiaya specimens exist. This area could be a new area of endemism, but spiders from this site are poorly or not found in collections and this area is poorly represented by governmental reserves and their remaining habitats are suffering high anthropic pressure as pointed by PINTO-DA-Rocha et al. (2005) for opilionid faunas.

Revista Brasileira de Zoologia 23 (2): 429-442, junho 2006 


\section{ACKNOWLEDGEMENTS}

We wish to thank Diana S. Davila, Cristina A. Rheims, and the two anonymous reviewers for helpful suggestions on the manuscript and Pedro Kyohara and Simone P. Toledo for making the scanning electron micrographs. This study was supported by CNPq (Conselho Nacional de Desenvolvimento Ciêntifico e Tecnológico) and FAPESP (Fundação de Amparo à Pesquisa do Estado de São Paulo, grant 99/05446-8 and 03/ 01058-0). This study is part of the BIOTA/FAPESP - The Biodiversity Virtual Institute Program (www.biotasp.org.br).

\section{REFERENCES}

BERTANI, R. 2001. Revision, cladistic analysis, and zoogeography of Vitalius, Nhandu, and Proshapalopus; with notes on other theraphosine genera (Araneae, Theraphosidae). Arquivos de Zoologia, São Paulo, 36: 265-356.

Bonnet, P. 1961. Bibliographia Araneorum. Tourlouse, Douladoure, $587 \mathrm{p}$.

Brescovit, A.D. 1996. Revisão do gênero Centroctenus Mello-Leitão (Araneae, Ctenidae, Cteninae). Revista Brasileira de Entomologia, São Paulo, 40 (2): 301-313.

Brignoli, P.M. 1983. A Catalog of the Araneae. Manchester, Manchester University Press, 755p.

Bryant, E.B. 1942. Additions to the spider fauna of Puerto Rico. Journal of Agriculture of the University of Porto Rico, Rio Piedras, 26: 1-16.

Griswold, C.E. 1991. Cladistic biogeography of Afromontane spiders. Australian Systematics in Botany, Sydney, 4: 7389.

Griswold, C.E. \& J. Ledford. 2001. A monograph of the migid trap door spiders of Madagascar and review of the world genera (Araneae, Mygalomorphae, Migidae). Occasional Papers of the California Academy of Sciences, San Francisco, 151: 1-120.

Lehtinen, P.T. 1967. Classification of the Cribellate spiders and some allied families, with notes on the evolution of the suborder Araneomorpha. Annales Zoologici Fennici, Helsinki, 4 (3): 199-468.

Received in 12.VIII.2005; accepted in 02.V.2006.
LOURENÇO, W.R. 1986. Les modeles de distribution geographique de quelques groupes de scorpions neotropicaux. Compte Rendu Sommaire des Séancesde la Societe de Biogéographie, Paris, 62: 61-83.

Mello-Leitão, C.F. DE 1915. Alguns gêneros e espécies novas de Araneidos do Brasil. Broteria, Rio de Janeiro, 13 (3): 129142.

MelLo-Leitão, C.F. DE. 1936. Contribution à l'étude des ctenides du Brésil. Festschrift Zum 60 Geburstage von Professor Dr. Embrik Strand, Riga, 1: 1-31.

Petrunkevitch, A. 1928. Systema Aranearum. Transactions of the Connecticut Academy of Arts and Sciences, New Haven, 29: 1-270.

PinTO-DA-RochA, R. 2002. Systematic review and cladistc analysis of the Brazilian subfamily Caelopyginae (Opiliones: Gonyleptidae). Arquivos de Zoologia, São Paulo, 36 (4): 357-464.

Pinto-da-Rocha, R.; M. B. Da Silva \& C. Bragagnolo. 2005. Faunistic similarity and historic biogeography of the harvestmen of Southern and Southeastern Atlantic Rain Forest of Brazil. Journal of Arachnology, Denver, 33 (2): 290-299.

Platnick, N.I. 1976. Drifting spiders or continents?: vicariance biogeography of the spider subfamily Laraniinae (Araneae: Gnaphosidae). Systematic Zoology, London, 25 (2): 101109.

PlatNick, N.I. 2005. The world spider catalog, version 3.5. American Museum of Natural History. On line at http:// research.amnh.org/entomology/spiders/catalog81-87/ index.html (Access in 27.V.2005).

SILVA, D. 2003. Higher-level relationships of the spider family Ctenidae (Araneae: Ctenoidea). Bulletin of the American Museum of Natural History, New York, 274: 1-86.

Simó, M. \& V.R.D. von EIckstedt. 1994. Revision de la sistematica del genero Asthenoctenus Simon, 1897 (Araneae, Ctenidae). Aracnología, Montevideo, 22/23: 1-12.

Tourinho-Davies, A.L. 2004. A new genus of Gagrellinae from Brazil, with a comparative study of some of the subtropical and southernmost tropical South American genera (Opiliones, Eupnoi, Sclerosomatinae). Revista Ibérica de Aracnología, Zaragosa, 9: 157-177. 